\title{
Pair distribution function and structure factor of spherical particles
}

\author{
Rafael C. Howell* \\ Materials Science and Technology Division, Los Alamos National Laboratory, Los Alamos, New Mexico 87545, USA \\ Thomas Proffent \\ Lujan Neutron Scattering Center, Los Alamos National Laboratory, Los Alamos, New Mexico 87545, USA \\ Steven D. Conradson团 \\ Materials Science and Technology Division, Los Alamos National Laboratory, Los Alamos, New Mexico 87545, USA
}

(Dated: February 2, 2008)

\begin{abstract}
The availability of neutron spallation-source instruments that provide total scattering powder diffraction has led to an increased application of real-space structure analysis using the pair distribution function. Currently, the analytical treatment of finite size effects within pair distribution refinement procedures is limited. To that end, an envelope function is derived which transforms the pair distribution function of an infinite solid into that of a spherical particle with the same crystal structure. Distributions of particle sizes are then considered, and the associated envelope function is used to predict the particle size distribution of an experimental sample of gold nanoparticles from its pair distribution function alone. Finally, complementing the wealth of existing diffraction analysis, the peak broadening for the structure factor of spherical particles, expressed as a convolution derived from the envelope functions, is calculated exactly for all particle size distributions considered, and peak maxima, offsets, and asymmetries are discussed.
\end{abstract}

\section{LA-UR 05-8264}

PACS numbers: 61.10.Dp, 61.12.Bt, 61.46.-w, 36.40.-c

\section{INTRODUCTION}

There currently exists much scientific interest of the physical and chemical properties of nanoparticles and nanodomains. Generally investigations of particles with diameters of the order of one micron are limited to powders, and from the diffraction data one performs Rietveld refinement to extrapolate their structure and size, by using the Debye-Scherrer formula, $\frac{1}{1}$ extensions such as Debye function analysis,, 2 and small-angle scattering, 3 Alternatively one derives the pair distribution function (PDF) from the diffraction data,,$\frac{4}{-}$ thus facilitating the study of size, correlated atomic motion,,$\frac{5}{5}$ short- to medium-range order,, 6 and other phenomena more apparent with a real-space treatment.

A number of techniques have been developed to infer the size and structure of particles from the PDF alone. For example, the local structure has been determined by an interpretation of the first peaks of the $\mathrm{PDF}, \frac{7}{-}$ and the size has been estimated from a Fourier transform of the wide-angle Debye-Scherrer diffraction pattern 8 In this manuscript, a rigorous approach to the determination of particle size is taken by rederiving the pair distribution function of a single spherical particle, expressed as an envelope function that multiplies the PDF of an infinite crystal with the same crystal structure ${ }^{\frac{9}{}}$ A general class of distributions of particle sizes is then considered, and an associated distributed envelope function is obtained. Using experimental PDFs of both bulk gold and gold nanoparticles, calculated from high- $Q$ neutron-powderdiffraction data, a distributed envelope function is used to transform the PDF of bulk gold to give a best fit repli- cation of the PDF of gold nanoparticles. Based on the parameters of this envelope function, the particle size distribution of the gold nanoparticles is predicted and compared to that obtained experimentally. Finally, a relationship between the real-space envelope function and a $Q$-space convolution function is established, the latter of which is to be applied to the structure factor of an infinite crystal to obtain that of a distribution of spherical particle sizes. The analytical form of the convolution function allows for a quantitative analysis of Bragg peak maxima, widths, and asymmetries as a function of peak position and particle size distribution. A thorough account of the relationship between the PDF and structure factor can be found in the literature 3.10

The formalism used is identical to Peterson et al. 11 with comparisons to other definitions and nomenclature found in Keen ${ }^{12}$ With little effort, the conclusions made here regarding the PDF and structure factor of spherical particles can also be carried over to embedded spherical domains, with the stipulation that the individual domains be uncorrelated with each other (i.e., they have random orientations), and uncorrelated with the host matrix. Indeed, diffraction analysis using spherical geometries has already been succcessful in studies of water in mesopores and micropores, 13 as well as distributions of particle and void sizes in NMR cryoporometry. 14,15 The structure factor analysis of spherical domains with well-defined atomic structures fits within the more general context of disorder within crystals, thus contributing to the analytical treatment of the associated diffuse scattering 16.17 .18 It also provides a means of quantifying the diffraction limit with respect to localized lattice dis- 
tortions, with spherical domains being a special case to be considered within the large class of nanoscale heterogeneities already studied. 19

\section{THE PAIR DISTRIBUTION FUNCTION OF A SINGLE SPHERICAL PARTICLE}

The microscopic pair density gives a distribution of atomic pair distances $r$ in a sample, weighted by the pair's scattering lengths

$$
\rho(r)=\frac{1}{4 \pi r^{2} N} \sum_{i \neq j} \frac{b_{i} b_{j}}{\langle b\rangle^{2}} \delta\left(r-r_{i j}\right)
$$

The following method of constructing $\rho(r)$ will be useful in determining its form with respect to spherical particles. For each $r$, define a spherical shell with this radius. Let the center of this sphere coincide with the position of an atom $i$, and record as weighted $\delta$ functions every atom $j$ that intersects the spherical shell. Finally, divide the result by its surface area and the total number of atoms $N$. The calculation of $\rho(r)$ for a spherical particle limits the position of the center of the sphere to the atoms within the particle itself, whereas the sphere's surface can extend beyond. Note that $\rho(r)$ for a single particle may only be a part of the total microscopic pair density of a solid or solution.

The contribution to $\rho(r)$ of all atomic pairs $\{i j\}$ within a spherical particle is limited to the range $0<r_{i j}<2 R$, where $R$ is its radius. If the particle is in solution, or we consider an ensemble of identical particles with random orientations embedded within a host lattice, then $\rho(r)=\rho_{0}$ for $r>2 R$, where $\rho_{0}$ is the constant atomic number density outside the particle. To simplify the following, let $\rho_{0}$ be equal to the number density of the particle itself, or take $\rho_{0}=0$ for empty space. The essence of the problem addressed hereafter is to quantify the relative population of atomic pair distances between any two atoms within the particle and pair distances where one atom resides outside of the particle. That is, $\rho(r)$ will have $r$-dependent contributions from both the microscopic pair density $\rho_{c}(r)$ of an infinite crystal and the uncorrelated outside structure $\rho_{0}$,

$$
\rho(r)=f_{e}(r, R) \rho_{c}(r)+\left[1-f_{e}(r, R)\right] \rho_{0},
$$

where $0 \leq f_{e}(r, R) \leq 1$. When $R \rightarrow \infty, f_{e}(r, R)=1$ for all $r$ and $\rho(r)$ is that of an infinite crystal. When $R \rightarrow 0, f_{e}(r, R)=0$ for all $r$ and $\rho(r)=\rho_{0} . f_{e}(r, R)$ is the envelope function that we now derive when $R$ is between these limits.

Consider a point within the particle whose position is given by the vector $\mathbf{r}^{\prime}$, with the center of the particle defining the origin. Orient the point and the particle so that $\mathbf{r}^{\prime}$ aligns with the $z$ axis, as shown in Fig. 11 A spherical shell of radius $r$ around this point will either be enclosed by the particle if $r<R-r^{\prime}$, intersect the

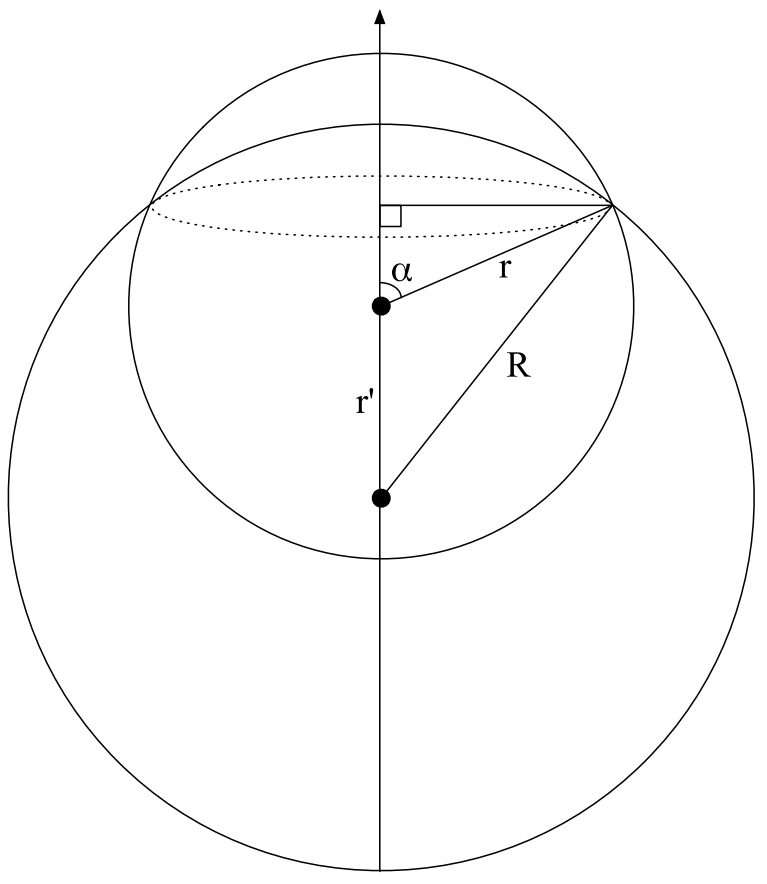

FIG. 1: A spherical particle with radius $R$. An atom a distance $r^{\prime}$ from the center of the particle can have a shell of radius $r$ that is only partially embedded within the particle.

surface of the particle if $R-r^{\prime} \leq r \leq R+r^{\prime}$, or enclose the particle if $r>R+r^{\prime}$.

When $R-r^{\prime} \leq r \leq R+r^{\prime}$, a line from any point on the circle of intersection and the position $r^{\prime}$ will meet the $z$ axis at an angle $\alpha$. The fraction of the surface of radius $r$ around this point that is enclosed within the particle is

$$
\begin{aligned}
f\left(r^{\prime}, r, R\right) & =\frac{1}{4 \pi r^{2}} \int_{0}^{2 \pi} r d \phi \int_{\alpha}^{\pi} r \sin \theta d \theta \\
& =\frac{1}{2}(1+\cos \alpha) .
\end{aligned}
$$

Using the two right triangles from Fig. 1] the angle $\alpha$ can be expressed as

$$
\cos \alpha=\frac{R^{2}-r^{\prime 2}-r^{2}}{2 r^{\prime} r} .
$$

The contribution of all such spheres enclosed within the particle is obtained by integrating $f\left(r^{\prime}, r, R\right)$ over the remaining positions $r^{\prime}$ in the region occupied by the particle, taking care to consider when the shell of radius $r$ extends outside the region. Using Eq. 固 for $r \leq 2 R$,

$$
\begin{aligned}
f(r, R) & =\frac{4 \pi}{3}(R-r)^{3}+4 \pi \int_{R-r}^{R} f\left(r^{\prime}, r, R\right) r^{\prime 2} d r^{\prime} \\
& =\frac{4 \pi}{3} R^{3}\left[1-\frac{3}{4} \frac{r}{R}+\frac{1}{16}\left(\frac{r}{R}\right)^{3}\right] .
\end{aligned}
$$

Finally, dividing by the total particle volume gives the envelope function

$$
f_{e}(r, d)=\left[1-\frac{3}{2} \frac{r}{d}+\frac{1}{2}\left(\frac{r}{d}\right)^{3}\right] \Theta(d-r),
$$


where $d=2 R$ is the particle diameter and $\Theta(x)=0(1)$ for negative (positive) $x$ is the Heaviside step function. $f_{e}(r, d)$ and its derivative are continuous for all positive $r$.

The PDF of the particle is related to the microscopic pair density

$$
\begin{aligned}
G(r, d) & =4 \pi r\left[\rho(r)-\rho_{0}\right] \\
& =f_{e}(r, d) G_{c}(r),
\end{aligned}
$$

where $G_{c}(r)=4 \pi r\left[\rho_{c}(r)-\rho_{0}\right]$ is the PDF of an infinite crystal with the same crystal structure as the particle.

The fraction of atom pairs residing within a spherical region is obviously not a continuous function of the region's size, considering the discrete nature of a crystal. Therefore, one expects the derivation above to become less accurate for smaller particle sizes. To test this, the exact calculation of $G(r)$ [using Eq. 1 for spherical particles with an ideal fcc structure with lattice constant $a$ was compared to $G(r)$ obtained by applying Eq. [7 to the PDF of an infinite ideal fcc structure. A particle was constructed with $n$ shells of atoms around a central atom, giving it a diameter of $d=\sqrt{2 n} a$ (some shells contain no atoms). While the difference in the nearest-neighbor peak heights between the two was $7 \%$ for $d=2 a$, the difference between all peaks rapidly diminished to less than $1 \%$ for $d=4 \sqrt{2} a$, and became negligible thereafter.

\section{DISTRIBUTIONS OF SPHERICAL PARTICLE SIZES}

The envelope function $f_{e}(r, d)$ depends on a singleparticle diameter $d$. From a distribution $P\left(d^{\prime}\right)$ that defines an ensemble of particle diameters $d^{\prime}$, a distributed envelope function $f_{\mathrm{DE}}(r)$ can be constructed by weight- ing individual single particle envelope functions with this distribution,

$$
f_{\mathrm{DE}}(r)=\int_{0}^{\infty} f_{e}\left(r, d^{\prime}\right) P\left(d^{\prime}\right) d d^{\prime}
$$

Consider the following normalized distribution:

$$
P\left(d^{\prime}, D, n\right)=\frac{1}{n ! D}\left(\frac{d^{\prime}}{D}\right)^{n} e^{-d^{\prime} / D}
$$

where $n$ is a positive integer and $D$ is a positive real number. The average particle diameter $d$ for this distribution is related to the parameters $n$ and $D$,

$$
d=\left\langle d^{\prime}\right\rangle=\int_{0}^{\infty} d^{\prime} P\left(d^{\prime}\right) d d^{\prime}=(n+1) D,
$$

and the characteristic width of the distribution is

$$
\sigma=\sqrt{\left\langle d^{\prime 2}\right\rangle-d^{2}}=\frac{d}{\sqrt{n+1}} .
$$

Hereafter the average diameter $d$ will be used to identify the associated distributed envelope functions and their behaviors, thus facilitating comparisons between those obtained from various distributions and the single particle size envelope function. The parameter $D$ is still used within function expressions, however, to maintain their simplicity. The two are always related by Eq. [10]

When deriving a distributed envelope function from Eqs. 8 and 9 alone, one recognizes that a weighted sum of these distributions (each with unique $n$ and $D$ ) yields a weighted sum of individual distributed envelope functions, so that the results hereafter can be easily adapted to a variety of distributions. For $n \geq 3$, a closed form expression of the resulting envelope function is given by

$$
f_{\mathrm{DE}}(r, d, n)=e^{-r / D} \sum_{k=0}^{n-2} \frac{1}{k !}\left(1-\frac{3}{2} \frac{k}{n}+\frac{1}{2} \frac{k(k-1)(k-2)}{n(n-1)(n-2)}\right)\left(\frac{r}{D}\right)^{k} .
$$

As with the single particle distribution $G(r, d, n)=$ $f_{\mathrm{DE}}(r, d, n) G_{c}(r)$.

Figure 2 shows the single particle size envelope function $f_{e}(r, d)$ (Eq. 6) and the distributed envelope functions $f_{\mathrm{DE}}(r, d, n)$ for $n=3$ and $n=9$ (expressed using the average diameters $d=4 D$ and $d=10 D$, respectively). The associated distributions are shown in the inset, and the case of $n=100$ is included to illustrate the trend of these distributions (the single particle size is a $\delta$ function distribution, and is not shown). The width of the distributions clearly dictates the shape of $f_{\mathrm{DE}}(r, d, n)$. A broader distribution gives the resulting envelope function a longer tail at large $r$ at the expense of a steeper decline for small $r$. Note that a universal condition for all spherical particle size distributions with average diameter $d$ is

$$
f(r=0, d)=1 \text { and } \int_{0}^{\infty} f(r, d) d r=\frac{3}{8} d .
$$

\section{COMPARISON WITH EXPERIMENT}

By comparing an experimentally obtained PDF of a collection of spherical-like particles with the theoretical expressions just derived, it should be possible to pre- 


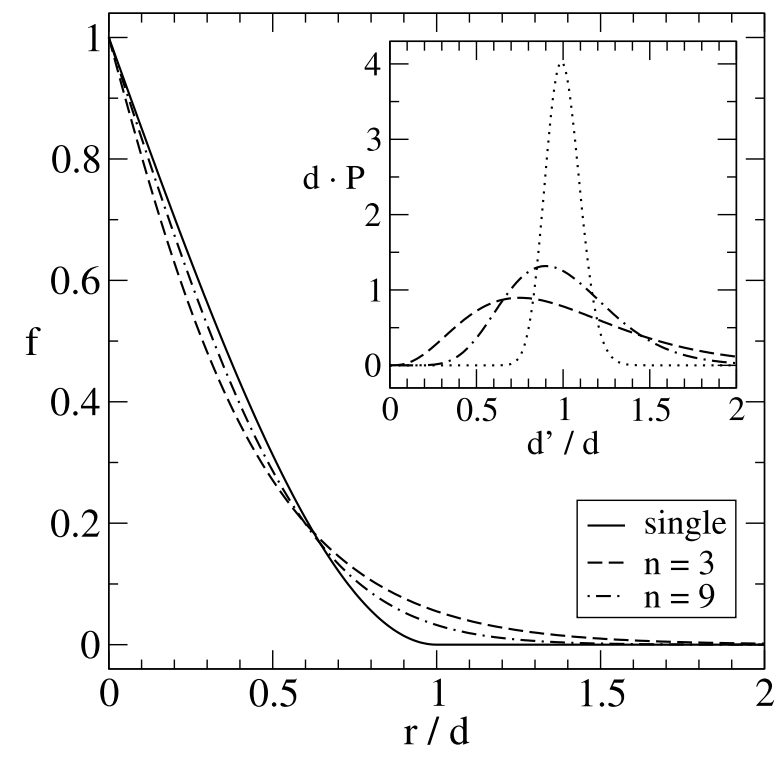

FIG. 2: The single particle size envelope function and two examples of distributed envelope functions, with $n=3$ and $n=9$. The associated distributions $P\left(d^{\prime} / d\right)$ are shown in the inset along with $n=100$ (dotted).

dict the particle size distribution used in the experiment. Neutron data of a $2 \mathrm{~g}$ batch of capped gold nanoparticles and a bulk gold fcc powder reference were collected at $T=15 \mathrm{~K}$ on the neutron powder diffractometer (NPDF) at the Lujan Center at Los Alamos National Laboratory 20 The PDF of both were obtained, and the bulk gold underwent full profile structural refinements, using PDFFIT, ${ }^{21}$ to account for a host of influences such as correlated and uncorrelated atomic motions and instrument resolution. Multiplying the refined PDF of the bulk data $G_{\mathrm{b}}(r)$ with an envelope function derived from either a single particle size $f_{e}(r, d)$ or a distribution of particle sizes $f_{\mathrm{DE}}(r, d, n)$ should give a good representation of the ideal PDF for spherical nanoparticles of fcc gold. A simplification is made by assuming that the crystal structure within the bulk gold and gold nanoparticles is identical. This simplification will be addressed when the results of the PDF comparisons are discussed.

Using the PDF data of gold nanoparticles $G_{\mathrm{np}}(r)$ the best fit diameter (and exponent for the distribution) is obtained by minimizing the root mean square deviation $\delta_{G}$ between this PDF and the transformed bulk PDF. The deviation is given by

$$
\delta_{G}^{2}=L^{-1} \int_{2.5}^{100.0}\left[G_{\mathrm{np}}(r)-f(r, d) G_{b}(r)\right]^{2} d r
$$

where the lower bound is chosen to ignore spurious oscillations in the experimental PDF, the upper bound is determined from the scattering resolution, and $L$ is the difference between these two. The integrations are performed numerically with a lattice spacing of $0.01 \AA$.

Table \compares the parameters from the best fit particle size distribution and the single particle distribution.

\begin{tabular}{lccrr}
\hline \hline Distribution & $\mathrm{n}$ & $\mathrm{d}(\AA)$ & $\sigma(\AA)$ & $\delta_{G}\left(\AA^{-2}\right)$ \\
\hline Single & & 28.75 & & 1.243 \\
$P\left(d^{\prime}, d, n\right)$ & \multirow{2}{*}{13} & 29.80 & 7.96 & 1.238 \\
Experiment & & 35.48 & 13.12 & \\
\hline \hline
\end{tabular}

TABLE I: A comparison of the parameters from the best fit particle size distributions and the experimentally determined distribution.

The predicted distribution of particle sizes, with $n=13$ and $d_{n}=29.80 \AA$, gives the smallest absolute deviation from the experimental PDF, with $\delta_{G}=1.238 \AA^{-2}$.

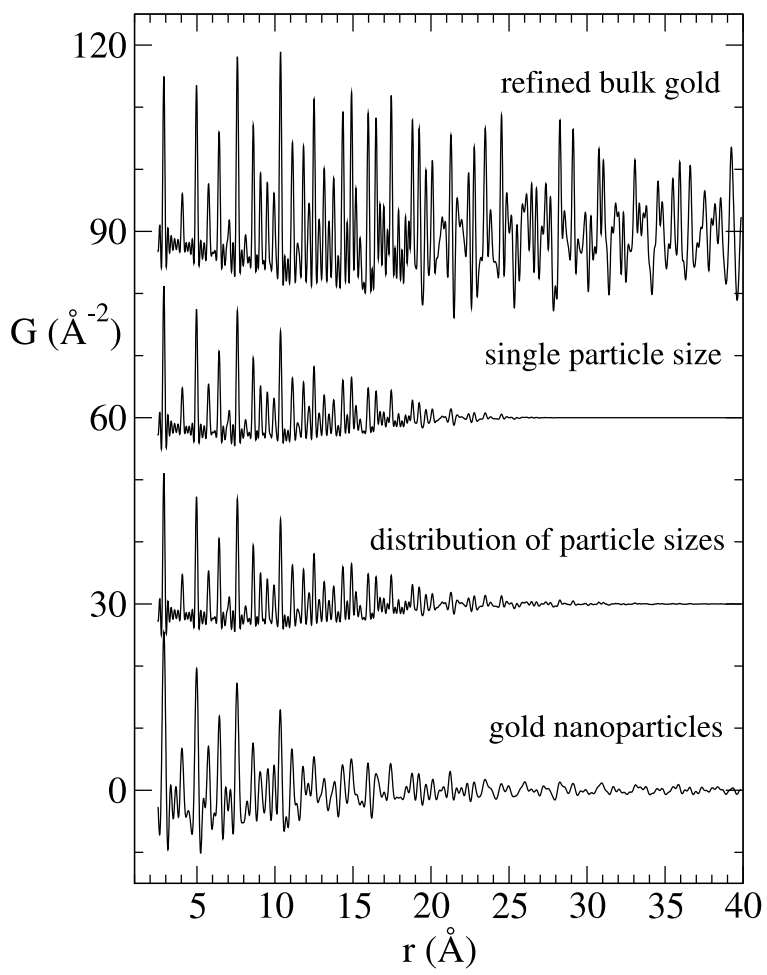

FIG. 3: An offset comparison of the refined experimental PDF from the bulk gold, the predicted PDF from the single particle size and the distribution of particle sizes, and the experimental PDF from the gold nanoparticles.

Figure 3 shows an offset comparison between the experimental PDF of the gold nanoparticles, the best fit PDFs from the single particle size and the distribution of particle sizes, and the refined PDF of the bulk gold used to obtain the fits. The envelope function for the distribution of particle sizes allows for correlations over longer atomic separations without changing the behavior of short-range correlations, as is evident in the figure, and is thus a better fit to the experimental PDF than that of the single particle size distribution. The PDF derived from a single envelope function always truncates to zero for $r \geq d$.

As a further test of the theory of spherical particles developed so far, a comparison of the particle size distri- 


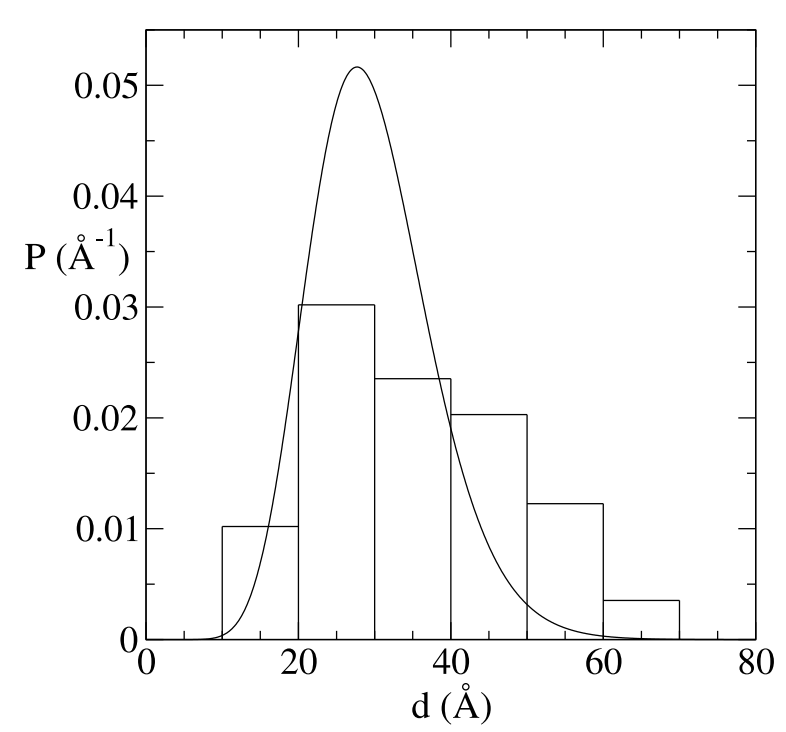

FIG. 4: A comparison of the experimentally determined particle size distribution (histogram), and the predicted particle size distribution from the best fit PDF.

bution given by minimizing Eq. 14 with the distribution obtained experimentally is now possible. Using a JEOL 2010 TEM with point-to-point resolution of $1.9 \AA$, the diameters of 148 gold nanoparticles within the experimental sample were measured, giving the distribution of particle sizes shown in Fig. 4 (histogram). The average particle diameter from this distribution is $d=35.48 \AA$, and the width of the distribution is $\sigma=13.12 \AA$. These values are given in Table【 Also shown in the figure is the predicted theoretical particle size distribution $(d=29.80$ $\AA, \sigma=7.96 \AA$ ). Obviously the best fit PDF predicts a distribution of particle sizes with a smaller average diameter and width. This can be attributed to the presence of pentagonal twinning seen within the gold nanoparticles (see Fig. 2 of Page et al ${ }^{20}$ ). The characteristic length scale of the twinned regions defines the scale of long-range order (an fcc structure in this case), which affects the resulting PDF. Each object might be better described as a collection of single domain crystallites instead of a spherical particle with a uniform structure. Equation 14 compares their PDF with the bulk gold PDF, in which twinning did not occur and the structure was uniform, and thus delivers a best fit envelope function that represents the size of the coherent fcc structure. The counting method used to construct the histogram in Fig. 团 however, considered only total particle size, which contributes to the discrepancy between the two distributions.

\section{THE STRUCTURE FACTOR OF SPHERICAL PARTICLES}

The total-scattering structure factor of spherical particles $S(Q, d)$ can be obtained directly from their PDF by a Sine transform

$$
\begin{aligned}
Q[S(Q, d)-1] & =\int_{0}^{\infty} G(r, d) \sin (Q r) d r \\
& =\int_{0}^{\infty} f(r, d) G_{c}(r) \sin (Q r) d r
\end{aligned}
$$

Let $S_{c}(Q)$ be the structure factor of the associated infinite crystal. $f(r, d)$ is an envelope function from any distribution of particle sizes, and can be expressed as the inverse cosine transform of a function $\bar{f}(Q, d)$,

$$
f(r, d)=\frac{2}{\pi} \int_{0}^{\infty} \bar{f}(Q, d) \cos (Q r) d Q .
$$

Since $G_{c}(r)$ is the inverse Sine transform of $Q\left[S_{c}(Q)-1\right]$, Eq. 15 can be written not only as a Sine transform of a product of two functions, but also as a convolution of their respective transforms ${ }^{22}$

$$
\begin{aligned}
Q(S(Q, d)-1)= & \frac{1}{\pi} \int_{0}^{\infty}\left[\bar{f}\left(\left|Q-Q^{\prime}\right|, d\right)-\bar{f}\left(Q+Q^{\prime}, d\right)\right] \\
& \times Q^{\prime}\left(S_{c}\left(Q^{\prime}\right)-1\right) d Q^{\prime}
\end{aligned}
$$

or

$$
\begin{aligned}
S(Q, d)= & \frac{1}{\pi Q} \int_{0}^{\infty}\left[\bar{f}\left(Q-Q^{\prime}, d\right)-\bar{f}\left(Q+Q^{\prime}, d\right)\right] \\
& \times Q^{\prime} S_{c}\left(Q^{\prime}\right) d Q^{\prime}
\end{aligned}
$$

The absolute value of the argument $Q-Q^{\prime}$ can be disregarded if one recognizes the convolution function $\bar{f}(Q, d)$ as an even function of $Q$.

For a single particle size distribution, the convolution function is

$$
\begin{aligned}
\bar{f}_{e}(Q, d) & =\int_{0}^{\infty} f_{e}(r, d) \cos (Q r) d r \\
& =\frac{3 d}{(Q d)^{2}}\left(n_{1}(Q d)+\frac{1}{(Q d)^{2}}+\frac{1}{2}\right),
\end{aligned}
$$

where $n_{1}(x)=-\cos (x) / x^{2}-\sin (x) / x$ is a spherical Bessel function of the second kind. $\bar{f}_{e}(Q, d)$ has a halfwidth at half-maximum of approximately $Q=3.48 / d$. This convolution function is very similar to the expression used for the intensity of scattering from randomly oriented identical spherical particles, and is often used in small-angle scattering analysis, ${ }^{3}$ but differs from the sinc function derived from a Fresnel construction of a spherical crystal that is often used to characterize powder diffraction lines.

The convolution function for the distribution of particle sizes defined by Eq. 9 can be obtained by noting that each term in Eq. 12 has a cosine transform proportional to 


$$
\int_{0}^{\infty} \frac{1}{k !}\left(\frac{r}{D}\right)^{k} e^{-\frac{r}{D}} \cos (Q r) d r=\frac{D}{\left[1+(Q D)^{2}\right]^{k+1}} \sum_{\substack{j=0 \\
j \text { even }}}^{k+1}(-1)^{j / 2}\left(\begin{array}{c}
k+1 \\
j
\end{array}\right)(Q D)^{j}
$$

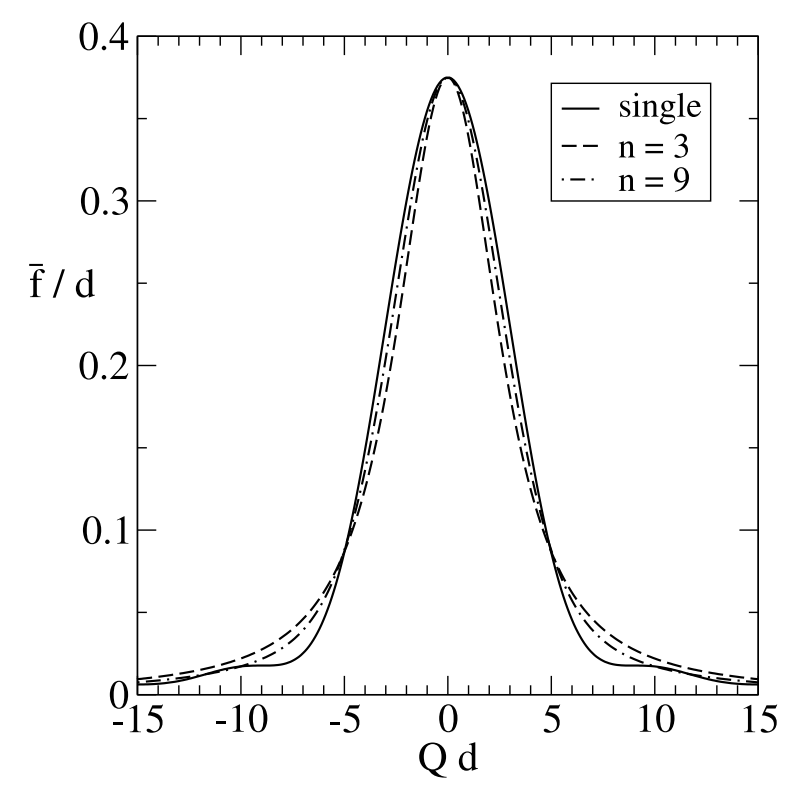

FIG. 5: The convolution functions to be applied to the infinite crystal structure factor $Q S_{c}(Q)$, for the single particle size and two distributions of sizes $n=3$ and $n=9$.

Figure 5 shows the convolution functions for the single particle size and the two distributions of sizes $n=3$ and $n=9$, considered before (expressed using the average diameters $d=4 D$ and $d=10 D$, respectively). For the single particle size, shoulders appear after the primary peak, a feature attributed to the similarity between a periodic envelope function and a triangular wave. When distributions of particle sizes are considered, the shape is always Lorentzian-like, due to the exponential behavior of the associated distributed envelope function.

A broader distribution gives the resulting convolution function a longer tail at large $Q$ at the expense of a steeper decline for small $Q$. Note that a corollary to Eq. $[13]$ is

$$
\bar{f}(Q=0, d)=\frac{3}{8} d \text { and } \frac{2}{\pi} \int_{0}^{\infty} \bar{f}(Q, d) d Q=1 .
$$

To summarize, the structure factor of a distribution of particle sizes can obtained by convoluting the structure factor of the associated infinite crystal with an envelope convolution function $\bar{f}(Q, d)$. The result will be a broadening of the Bragg peaks of the infinite crystal, but note that an asymmetry arises in this broadening, particularly for low $Q$, as Eq. 17 is actually the difference of two convolutions, each weighted by $Q^{\prime}$.

To illustrate this asymmetry, consider the effect that a convolution resulting from finite particle sizes has on a

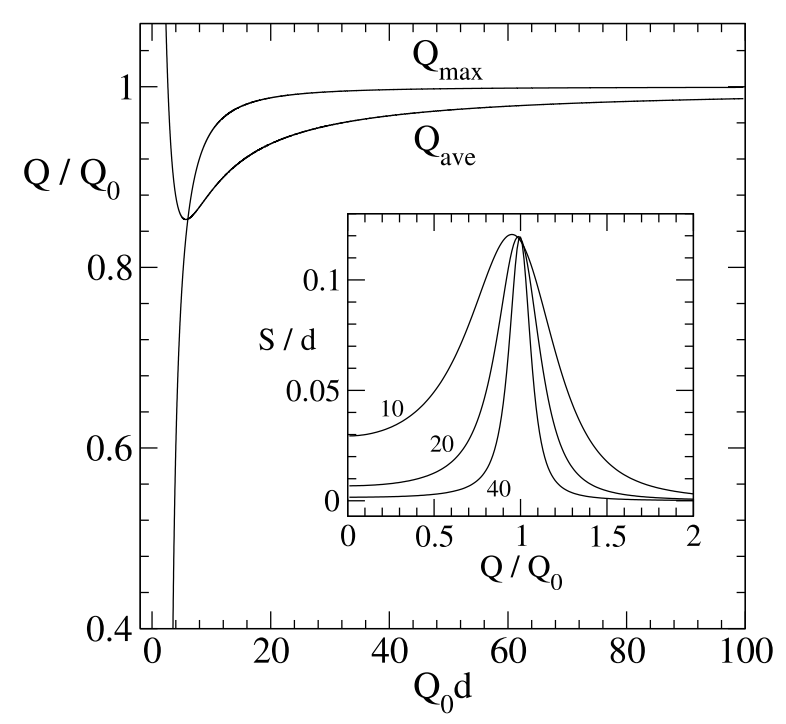

FIG. 6: A structure factor peak from spherical particles has a peak maximum and peak average below the ideal peak position $Q_{0}$. The asymmetries of the peak are illustrated in the inset for $Q_{0} d=10,20$, and 40 .

single ideal Bragg peak from an infinite crystal $S_{c}(Q)=$ $\delta\left(Q-Q_{0}\right)$, with the effects on all other ideal Bragg peaks being accounted for in a piecewise manner. A distribution of particle sizes with average diameter $d$ and exponent $n=3$ gives a convolution function (shown in Fig. 5)

$$
\bar{f}_{\mathrm{DE}}(Q, d)=2 d \frac{48+(Q d)^{2}}{\left[16+(Q d)^{2}\right]^{2}} .
$$

The convolution of the two [Eq. 17] produces a broadened peak for $S(Q)$, shown in the inset of Fig. [6 for $Q_{0} d=10$, 20 , and 40. A smaller average particle diameter $d$ (or a lower value of the peak position $Q_{0}$ ) transforms the ideal peak of $S_{c}(Q)$ more asymmetrically than a larger average particle diameter (or a higher peak position). The peak symmetry is restored for very large values of $Q_{0} d$, as the subtracted term in Eq. 17 becomes negligible.

Both the peak maximum $Q_{\max }$ and the average peak position (the normalized first moment) $Q_{\text {ave }}$ are also shown in the figure as a function of $Q_{0} d$. For most values of $Q_{0} d, Q_{\text {ave }}<Q_{\max }<Q_{0}$, which suggests that not only does the peak position shift to lower values of $Q$, but it also diminishes slower to the left of the peak maximum than to the right. For the extreme case when $Q_{0} d \lesssim 5.75$, $Q_{\max }$ quickly goes to zero, and as a result $Q_{\text {ave }}$ actually increases (it continues to broaden as $Q_{0} d$ decreases, but 
only for positive $Q$ ). This occurs when the characteristic width of $\bar{f}_{\mathrm{DE}}(Q, d)$ is approximately $Q_{0}$.

\section{CONCLUSION}

The predictive power of having an analytical form of the PDF of spherical particles has been clearly demonstrated. In this case we were provided with structurally refined experimental PDF data from a neutron source and a distribution of particle diameters observed from a TEM. Only the two together provided a means of testing the presented theory. With care, one could arrive at the same predictions by deconvoluting the experimental structure factor data of gold nanoparticles using Eq. 17 but the refinement analysis that afforded us a comparison between theory and experiment suggested a real-space treatment. Nevertheless, we believe that the convolution functions derived here can complement the peak shape analysis already used in modern Rietveld packages ${ }^{23}$ which often use Lorentzian and Gaussian functions alone to describe the peak broadening due to particle size effects. Ideally, the analytical form of the peak shapes, as derived from the convolution functions, should allow one to predict an entire particle size distribution, and not just an average particle size, by considering together the peak maxima, peak offsets, and peak asymmetries from an experimental structure factor.

It was mentioned earlier that the analysis of spherical particles should provide immediate insight into the effects of nanoscale domains embedded within a host lattice. When two structures coexist as uncorrelated domains, for example, a chemically disordered fcc structure and a second chemically ordered structure with a small tetragonal distortion, the total PDF of the solid can be taken as the sum of the individual PDFs, each calculated with a unique envelope function suitable to their differing domain sizes. The broadening of the Bragg peaks resulting from their finite size, combined with the peak splitting of the second tetragonal phase, may dictate whether the chemically ordered domains are below the diffraction limit, thus requiring a real-space probe such as XAFS to accurately measure their local structure. This analysis may be useful in explaining the presence of magnetism in NiMn alloys when there is no signature of $L 1_{0}$ ordering of the material apparent in the diffraction data. ${ }^{24}$

Finally, the real-space treatment of PDF analysis, and the subsequent conclusions made about the structure factor, is an encouraging approach to solving problems that are often only considered in $Q$ space. For example, the idea that a particle's surface might have a different structure than its core (internal strain), can be realized in real space by considering both an envelope function (for particle size), and a convolution function (for varying strain). Can the structure factor be derived from the two taken together, just as it was for the envelope function alone, or does this problem require the $Q$-space treatments already considered $?^{25}$

\section{Acknowledgments}

This work was supported by the Heavy Element Chemistry Program, Chemical Sciences, Biosciences, and Geosciences Division, Office of Basic Energy Sciences, and Defense Programs, NNSA, U.S. Department of Energy under Contract No. W-7405. It has also benefited from the use of NPDF at the Lujan Center at Los Alamos Neutron Science Center, funded by the DOE Office of Basic Energy Sciences, Los Alamos National Laboratory, and the Department of Energy under Contract No. W7405-ENG-36. The upgrade of NPDF has been funded by NSF through Grant No. DMR 00-76488.
* Electronic address: rhowell@lanl.gov

† Electronic address: tproffen@lanl.gov

¥ Electronic address: conradson@lanl.gov

1 P. Scherrer, Gött. Nachr. 2, 98 (1918).

2 W. Vogel, Cryst. Res. Technol. 33, 1141-1154 (1998).

3 A. Guinier, X-Ray Diffraction (Freeman, San Francisco, 1963).

4 T. Proffen, S. J. L. Billinge, T. Egami, and D. Louca, Z. Kristallogr. 218, 132 (2003).

5 I. Jeong, T. Proffen, F. Mohiuddin-Jacobs, and S. J. L. Billinge, J. Phys. Chem. A 103, 921 (1999).

6 T. Egami, J. Phys. Chem. Solids 56, 1407 (1995).

7 R. B. Neder and V. I. Korsunskiy, J. Phys.: Condens. Matter 17, S125 (2005).

8 B. D. Hall, D. Zanchet, and D. Ugarte, J. Appl. Crystallogr. 33, 1335 (2000).

9 L. H. Germer and A. H. White, Phys. Rev. 60, 447 (1941).

10 J. Waser and V. Schomaker, Rev. Mod. Phys. 25, 671 (1953).

11 P. F. Peterson, E. S. Bozin, T. Proffen, and S. J. L. Billinge,
J. Appl. Crystallogr. 36, 53 (2003).

12 D. A. Keen, J. Appl. Crystallogr. 34, 172 (2001).

13 D. C. Steytler, J. C. Dore, and C. J Wright, Mol. Phys. 48, 1031 (1983).

14 J. B. W. Webber, J. H. Strange, and J. C. Dore, Magn. Reson. Imaging 19295 (2001).

15 J.B.W. Webber, Ph.D. thesis, University of Kent, UK, (2000). Available online: http://www.kent.ac.uk/physical-sciences/publications/theses/jbww.ht

16 F. Frey, Z. Kristallogr. 212, 257 (1997).

17 G. E. Ice and C. J. Sparks, Annu. Rev. Mater. Sci. 29, 25 (1999).

18 C. N. J. Wagner, J. Non-Cryst. Solids 31, 1 (1978).

19 A. J. Garcia-Adeva, D. R. Conradson, P. Villella, and S. D. Conradson, J. Phys. Chem B 107, 6704 (2003).

${ }^{20}$ K. Page, T. Proffen, H. Terrones, M. Terrones, L. Lee, Y. Yang, S. Stemmer, R. Seshadri, and A. K. Cheetham, Chem. Phys. Lett. 393, 385 (2004).

21 T. Proffen and S. Billinge, J. Appl. Crystallogr. 32, 572 (1999). 
${ }^{22}$ F. B. Hildebrand, Advanced Calculus for Applications (Prentice-Hall, New Jersey, 1976).

23 A. Larson and R. Von Dreele, General Structure Analysis System (GSAS), Los Alamos National Laboratory, Report No. LAUR 86-748 (unpublished).

${ }^{24}$ F. J. Espinosa-Faller, R. C. Howell, A. J. Garcia-Adeva,
S. D. Conradson, A. Y. Ignatov, T. A. Tyson, R. F. C. Farrow, and M. F. Toney, J. Phys. Chem. B 109, 10406 (2005).

25 A. Borbély and I. Groma, Appl. Phys. Lett., 79, 1772 (2001). 